\title{
LA COMUNICABILIDAD DE LO AMBIGUO: UNA PROPUESTA NARRATOLÓGICA PARA EL ANÁLISIS DE LA FICCIÓN TELEVISIVA COMPLEJA
}

\author{
THE COMMUNICATIVENESS OF THE AMBIGUOUS: A NARRATO- \\ LOGICAL PROPOSAL FOR THE ANALYSIS OF COMPLEX TELE- \\ VISION FICTION
}

\author{
Antonio LORIGUILLO-LÓPEZ \\ Universitat Jaume I \\ loriguil@uji.es
}

Resumen: El presente artículo propone una metodología de análisis de la narración ambigua en la ficción televisiva contemporánea, rasgo estilístico al alza entre propuestas comerciales. A través de la adaptación y aplicación de la comunicabilidad enunciada por David Bordwell como estrategia narrativa en su estudio de los modos de narración cinematográficos a una muestra de series dramáticas de origen anglosajón, se ofrece un primer tratamiento del modo narrativo de lo que desde los estudios televisivos se ha identificado como televisión compleja. Finalmente, se apunta a la correspondencia de este modelo con una nueva fase del modelo de narración del audiovisual postclásico.

Palabras clave: Televisión. Narratología. Ambigüedad. Punto de vista. Policíaco. 


\begin{abstract}
The present article proposes a methodology of analysis of the ambiguous narration in contemporary television fiction, a stylistic feature on the rise among commercial titles. Through the adaptation and application of the communicativeness formulated by David Bordwell as one of the categories of narrative strategies in his examination of the modes of narration in film to a sample of drama series produced in English-speaking regions, we offer a primary approach to the narrative mode of what has come to be known in the field of Television Studies as complex TV. Lastly, we note the correspondence between this model and a new phase of the audiovisual post-classical narration.
\end{abstract}

Key Words: Television. Narratology. Ambiguity. Point of view. Police crime drama.

\title{
1. INTRODUCCIÓN
}

En las últimas décadas diversas investigaciones han tratado de hacerse cargo de un fenómeno emergente en el audiovisual contemporáneo: la irrupción de la complejidad narrativa en el circuito comercial. Concepto resbaladizo y, hasta cierto punto, discutible ${ }^{1}$, la complejidad asociada a la narración fílmica y televisiva está definida por diversos académicos como una consciente obscuridad u ofuscación de la coherencia en el desarrollo de los relatos en favor de un incremento en la dificultad de su lectura y comprensión. Si para dar sentido a un texto se requiere de una integración inteligible de sus elementos entre sí, una integración que implique una relación con modelos familiares de coherencia (ya sean derivados de la "realidad" o de formas literarias previas (Rimmon-Kenan, 2002: 123), la narración compleja tensa en extremo la homología - la correspondencia estructural entre las fábulas de las narraciones y las fábulas reales construida

\footnotetext{
${ }^{1}$ Nos permitimos a modo de licencia recuperar al poeta Richard O. Moore, "[s]implicities are enormously complex. Consider the sentence 'I love you'" (2010).
} 
conforme a las exigencias de la lógica natural de los acontecimientos para la experiencia humana (Bal, 1985: 20) — en la que se apoya la narrativa convencional. Esta tensión se basa en el ataque a tres de sus pilares esenciales: la cronología, la falsabilidad sistemática de la experiencia de los personajes focalizadores y la causalidad. La narración compleja es rica en anacronías homodiegéticas incompletas y, sobre todo, en acronías inmotivadas diegéticamente. En relación a las primeras es destacable la preferencia por la narración delegada en forma de "focalización interna" constante en narradores poco fiables (por su conocimiento limitado, pero también por su implicación personal y por una problemática escala de valores; Rimmon-Kenan, 2002: 101) y la sistemáticamente huida de la fiabilidad de la "focalización cero" genettiana (1989: 244-245).

Ante la complejidad narrativa, la desconfianza de los espectadores emerge como principal actitud al enfrentarse a los textos. Esta no es una operación radicalmente original. Los precedentes son muchos y van desde la ambigüedad narrativa de Otra vuelta de tuerca de Henry James a las "obras límite" (Genette, 1989: 91) de Robbe-Grillet. Lo reseñable es que, si bien antes esta previsión frente a los textos era cuantitativamente marginal y se restringía a obras calificadas como absurdas o experimentales, en la actualidad goza de una popularidad central como estrategia en creciente aplicación en el mainstream comercial. Primordialmente etiquetadas en los estudios fílmicos bajo las nomenclaturas "puzzle films" (Buckland, 2009) y "mind-game films" (Elsaesser, 2009), este tipo de producciones ha venido discutiendo desde la década de 1990 el Modo de Representación Institucional (Burch, 1987), el modelo narrativo dominante en el cine y la televisión comercial. Sus rasgos más evidentes — una estructura regida por una cadena causal que progresa linealmente hacia una inequívoca clausura (Marzal-Felici, 1994) - , herederos de la reelaboración que el melodrama burgués efectúa sobre la poética aristotélica (2004), siguen formando parte esencial de las maneras de narrar del audiovisual contemporáneo y de unos valores que se corresponden con la posición ideológica de la cultura hegemónica. Como resume Palao Errando (2004: 93): 
La idea de un Modelo de Representación, de fundamentación metonímica, que ejerza el papel de marco ontológico para la experiencia de un mundo pleno es inherente a la concepción cosmológica de la Modernidad Occidental. Sin ambages: el MRI [es] la traducción del Modelo Mecanicista del Universo al ámbito de la representación audiovisual; infinitud, contigüidad, homogeneidad e imposibilidad lógica del vacio son sus fundamentos para guiar a la experiencia del Ser por los caminos de la plenitud óntica.

En este artículo ponemos atención en las tensiones que la narración compleja ejerce sobre el Modo de Representación Institucional. Al contrario que los postulados de éste - parciales hacia la eliminación total de ambigüedades para potenciar la absoluta certeza de una cadena de causas y efectos fácil de seguir mediante la puesta en juego de emociones universales para los espectadores- la narración compleja pone en juego una serie de recursos que tensan sobremanera las convenciones del clasicismo narrativo. Loops temporales, focalizaciones reducidas y/o múltiples, puntos de vista contradictorios, finales alternativos o mecanismos de autoreferencialidad son algunos de los elementos que discuten la domesticación de los relatos en la jaula de la univocidad. El término "puzzle films" es especialmente afortunado para designar a unas películas que disponen su syuzhet a la manera de la caja recién abierta de un rompecabezas: elementos (des)ordenados de forma no lineal, mise en abîme no jerarquizadas entre sí y causalidad diluida en grandes lagunas de conocimiento sobre la diégesis. Como la persona que consigue completar un rompecabezas, los espectadores de un filme como Memento (Nolan, 2000) pueden vanagloriarse de encajar todas las piezas de su trama. Aunque la complejidad narrativa no es, a priori, afín al disfrute cinematográfico convencional — dado a la instrumentalización de la ficción como ventana a otras experiencias y emociones, o como organizador (y sancionador) 
de los límites de la experiencia individual - su existencia en el seno de notables ejemplos del cine comercial contemporáneo es ilustrativa de una transición desde la pasividad espectatorial del clasicismo hacia una más consciente implicación por parte de las audiencias, muchas veces a través "epistemological problems (how do we know what we know) and ontological doubts (about other worlds, other minds)"2 (Elsaesser, 2009: 15). Como indica Warren Buckland (2014: 5):

these puzzle films challenge deep-seated cultural conventions that regulate and try to stabilize consciousness and representation, plus the attendant concepts that support these conventions (agency, discrete identity, memory, temporal linearity, singularity, etc. $)^{3}$.

Aunque la historiografía de la narración compleja se ha articulado en torno al cine ${ }^{4}$, las series dramáticas contemporáneas presentan un creciente número de textos susceptibles de ser estudiados bajo el paradigma de la narración compleja. Superando el determinismo tecnológico que subestimó las capacidades del medio televisivo para la transmisión de historias ${ }^{5}$,

\footnotetext{
${ }^{2}$ Traducción del autor (en adelante T. d. a.): “problemas epistemológicos (cómo sabemos lo que sabemos) y dudas ontológicas (sobre otros mundos, otras mentes)".

${ }^{3}$ T. d. a.: "estas puzzle films desafían las arraigadas convenciones culturales que regulan y tratan de estabilizar consciencia y representación, además de desafiar conceptos concomitantes que apoyan dichas convenciones (agencia, identidad discreta, memoria, linealidad temporal, singularidad, etc.)".

${ }^{4}$ Damos cuenta de lo prolífico de estas investigaciones por la variedad de nomenclaturas generadas alrededor del mismo fenómeno: "multiple draft films" (Branigan, 2002), "database narratives" (Kinder, 2002), "mindfuck movies" (Eig, 2003), "complex narratives" (Klinger, 2006 y Simons, 2008), "modular narratives" (Cameron, 2008), "forking-path narratives" (Bordwell, 2002), "mind-tricking narratives" (Klecker, 2013), "puzzle pictures" (Sterritt, 2016).

${ }^{5}$ La televisión era para McLuhan un medio frío, "un plano mosaico bidimensional" (1995: 273), por su parca resolución de imagen en comparación con el cine o la fotografía. Actualmente argumentos como el de McLuhan han sufrido un importante revés en el plano tecnológico, ya que las proyecciones domésticas mediante sistemas Home Cinema, capaces incluso de proyectar en estereoscopía 3D compiten en resolución con las micro-
} 
la historiografía de la ficción televisiva localiza los hitos que ayudan a contextualizar la aparición de la televisión compleja. En paralelo al desarrollo de las "puzzle films" en el seno del cine independiente estadounidense, los ochenta traen cambios estructurales importantes en los que es necesario detenernos para contextualizar la aparición de la narración compleja en televisión. El proceso de cambio, conocido por algunos investigadores como "TVII" (Creeber, 2013: 51), está representado por el cambio de tendencia de un modelo de broadcasting hacia el "narrowcasting": la producción de contenido no para su retransmisión generalista, sino para la multitud de nuevos canales especializados que surge tras la extensión de la televisión por cable y las retransmisiones vía satélite.

La fragmentación de la oferta de la ficción televisiva en esta época, alejada del maniqueísmo entre los canales públicos y comerciales, es junto a otros factores ${ }^{7}$ el caldo de cultivo para el surgimiento de unos canales con producción propia que, sustentados por un número determinado de suscriptores, operan con un cierto margen en el delicado balance entre la viabilidad comercial y el retorno de la inversión. De entre los productos que emergen de este contexto destaca, junto al descubrimiento del reality show, las series dramáticas con narración compleja. El caso de HBO es paradigmático de su explotación por ser la network de cable referente en cuanto a romper tabús y a consolidar la complejidad narrativa y la incorrección política en la ficción televisiva comercial (Leverette, 2008). Como indica Concepción Cascajosa Virino, se trata de "contenidos tabú en televisión que iban también a permitir atraer a un público joven y a crear una imagen de marca" (2005: 101-102) — tanto para los canales implicados

salas de exhibición comercial.

${ }^{6} \mathrm{Al}$ considerarlo una fase de disponibilidad de programas televisivos frente a la escasez del periodo formativo de la televisión, etiquetado como "TVI".

${ }^{7}$ Otras innovaciones tecnológicas, aquellas que tienen que ver con la mejora de equipos e integración de lo digital en las rutinas de producción televisivas, son capitales para títulos con una puesta en escena no convencional. Por ejemplo, Creeber se refiere al auge del estilo naturalista gracias a los rodajes fuera de plató con cámaras y equipos más ligeros y a la introducción de efectos visuales en postproducción (2013: 52). 
como para los showrunners, considerados como verdaderos autores en el s. XXI (Hernández Pérez, 2013) — consolidada a su vez en las últimas décadas por series apreciadas por crítica y público por su estilo y reflexión sobre las equívocas acepciones de la American way of life (Rubio Alcover, 2011 y Hernández Pérez y Revert Gomis, 2016) como Breaking Bad (Gilligan, AMC: 2008-2013) o Mad Men (Weiner, AMC: 2007-2015).

Pese a su evidente notoriedad, esta narración compleja, aparentemente indiscutible por ser celebrada al unísono por crítica y academia, no cuenta con una definición precisa anclada en fundamentos de la narratología audiovisual, algo paradójico si se tiene en cuenta que para las publicaciones divulgativas e investigadoras este rasgo ocupa un lugar central en las ventajas competitivas que estos textos presenten en el entorno de la sobreoferta del entretenimiento contemporáneo. El propósito de nuestra aportación es el de ofrecer un modelo de análisis de base narratológica de la televisión compleja. Para ello, aplicamos las categorías de estrategias narrativas desarrolladas por David Bordwell (con especial atención a la comunicabilidad) en su poética de los modos de narración del cine (1996) —actualizados al contexto del audiovisual postclásico televisivo mediante con las contribuciones teóricas de Eleftheria Thanouli (2009), Jason Mittell (2006) y Christine Piepiorka (2013) — a un corpus limitado de esta tendencia en el contexto de las series televisivas dramáticas estadounidenses y británicas, las más representativas por su impacto global.

\section{ESTADO DE LA CUESTIÓN. REVISIÓN CRÍTICA DE LAS APORTACIONES TEÓRICAS SOBRE COMPLEX TV}

En esta investigación tenemos como objetivo expandir el terreno que diversos autores han abierto en el multidisciplinar campo de los estudios sobre televisión, siempre siendo conscientes del delicado equilibrio metodológico que ofrece la conciliación de los aspectos semióticos y los de la economía política. Como apunta Jim Collins (1992: 255): 
[t]he problem for television studies, as it tries to come to terms with postmodernism, is how to reconcile the semiotic and economic dimensions of television. Stressing the semiotic to the exclusion of the economic produces only a formalist game of "let's count the intertexts," but privileging the economic to the point that semiotic complexity is reduced to a limited set of moves allowed by a master system is just as simplistic ${ }^{8}$.

La evidente transformación en la manera en la que se cuentan las historias durante los últimos años explica la eminencia de la consabida complejidad narrativa como elemento inherente a la conocida como tercera edad dorada de la televisión. Como apunta Jonathan Bignell (2007: 170):

Popular television series rely on recurrent narrative patterns where, as Umberto Eco argued, formulas produce pleasure for the viewer by rewarding predictive activity. So the pleasures of a specific narrative, such as setting up an enigma that will subsequently be resolved, produce a second kind of pleasure at the level of the series as a whole through repetition of narrative patterns and the programme's conformity to viewer expectations. Those US series considered quality television work with an economy comprising generic verisimilitude's adaptation of programmes to audience expectation, and also play with verisimilitude and genre by means of visual pleasure and spectacle. $A$ reflexive awareness that these programmes are television is crucial to their play with contrasts between excessive or uncon-

\footnotetext{
${ }^{8}$ T. d. a.: "el problema de los television studies es, mientras trata de acercar posturas con el postmodernismo, cómo reconciliar las dimensiones semióticas y económicas de lo televisivo. Favorecer lo semiótico frente a lo económico lleva únicamente al juego formalista de 'vamos a contar intertextos', pero privilegiar lo económico hasta el punto de que la complejidad semiótica es reducida a un repertorio de movimientos limitados permitidos por un sistema maestro es igual de simplista".
} 
ventional mise-en-scène and generic narrative, characterisation and dialogue .

De entre las investigaciones dedicadas a la historia del medio en el nuevo milenio destacan las publicaciones de Jason Mittell, pionero en el uso del término "narrative complexity" en aproximaciones históricas a la ficción televisiva: "[a] model of television storytelling distinct for its use of narrative complexity as an alternative to the conventional episodic and serial forms that have typified most American television since its inception" (2006: 29). Las aportaciones de Mittell tratan algunas de las diversas acepciones de dicha complejidad. Su tratamiento de la relación entre la ambigüedad narrativa y las comunidades virtuales de fans (2009), de las innovaciones en maneras de consumir televisión (2011), así como de la emergencia del antihéroe en los argumentos (2014) y los influjos de los videojuegos en la narrativa televisiva (2017), constituyen la base sobre la que se articulan posteriores investigaciones de cariz multidisciplinar sobre narrativas transmedia, sobre la metaficción en la cultura popular o sobre la identificación espectatorial con personajes turbados. Su contribución influyente y continuada a la teorización de la complejidad televisiva cristaliza en su texto Complex TV: The Poetics of Contemporary Television Storytelling (2015). En su ambiciosa empresa de aprehender una poética de las series narrativamente complejas, Mittell dedica epígrafes a la

\footnotetext{
${ }^{9}$ T. d. a.: "Las series televisivas populares se apoyan en patrones narrativos recurrentes donde, como Umberto Eco defendía, las fórmulas premian al espectador con el placer de la actividad predictiva. De esta manera, los placeres de una narrativa específica, como el establecimiento de un enigma que será sucesivamente resuelto, produce un segundo tipo de placer a nivel de la serie como un todo a través de la repetición de patrones narrativos y de la conformidad del programa a las expectativas de los espectadores. Esas series norteamericanas consideradas televisión de calidad trabajan con una economía que comprende la verosimilitud genérica de la adaptación de los programas a las expectativas de la audiencia, y también juegan con la verosimilitud y el género como medio del placer visual y del espectáculo. La consciencia reflexiva de que estos programas son televisión es crucial para sus juegos con los contrastes entre la puesta en escena excesiva o poco convencional y la narrativa genérica, la caracterización y el diálogo".
} 
práctica totalidad de lo que denomina "complex TV": la similitud entre showrunner y el auteur, a lo melodramático de la serialidad, a la construcción de personajes ambiguos, a la proliferación de fancition y a los relatos cross-media. Pese a su relevancia capital para los estudios televisivos, las observaciones que Mittell ha realizado sobre series de contrastada complejidad como Perdidos (Lieber, Abrams y Lindelof, ABC: 2004-2010) o Breaking Bad adolecen de un análisis detallado de las estrategias narrativas que, como el propio Mittell expone, son su atributo más distintivo con respecto al resto de oferta del entretenimiento televisivo. El estudio de Mittell trata tanto la diégesis como los condicionantes productivos, pero éstos, como la difusión transmediática o los paratextos fans, no son apuntalados por un comentario crítico sobre la narración, recurso propio de la "complex TV" en su definición.

La investigadora Christine Piepiorka (2013) deja atrás descripciones argumentales y actividad fan para enfocar el interés en la monitorización de un cambio en la estructura dramática de la ficción televisiva. Para ello, amplía el concepto de complejidad de Mittell para realizar una tipología estructural de la ficción televisiva desde la narratología (Tabla 1). Piepiorka distingue así entre series — asimilables a las antologías de episodios autoconclusivos que no requieren respetar un orden de visionado cronológico-, seriales — los más habituales por el desarrollo abierto de líneas argumentales de protagonismo coral mediante arcos dramáticos sucesivos - y seriales complejos. Estos seriales complejos son representativos de las intenciones de productores y creadores de hacer complejas las series televisivas mediante la conocida como multitrama ("multithreading"): la congregación de una gran cantidad de líneas argumentales en un mismo episodio. Como ilustra Piepiorka con el paradigmático caso de Perdidos (2013: 188-190):

In episodes one to six of season one, Lost develops thirty-three story arcs, while episode four alone consists in nine of them. Only two of those are introduced and closed in that episode [...] 
serials like How I Met Your Mother (USA 2005-, CBS) also exhibit interwoven elements-even though they emerge more from details than from whole story arcs (like, for example, the yellow umbrella of the kids'mother, which is mentioned again and again like a golden thread of the story). And if that was not enough: Narrative complexity is constituted by the fact that some narrative connections are first shown in the first episode and not finished until the last episode of the same season. Story arcs do not only skip one season, they even skip seasons ${ }^{10}$.

\begin{tabular}{c|ccc}
\hline & Distribución de la diégesis & Progresión dramática & $\mathbf{N}^{\circ}$ líneas argumentales \\
\hline Series & Narraciones episódicas & Lineal & Una \\
Seriales & Arcos dramáticos & Lineal & Varias \\
Seriales complejos & Arcos dramáticos no consecutivos & No lineal & Varias
\end{tabular}

Tabla 1. Tipología de la ficción televisiva según Piepiorka (2013). Fuente: elaboración propia.

Bajo nuestro punto de vista, la construcción multitrama es a las series de televisión contemporáneas lo que el modelo de narración postclásico es al cine comercial de hoy: el nuevo estándar narrativo de las producciones comercial actuales. Aunque la información por episodio aumente notablemente y se obligue al espectador a prestar máxima atención

\footnotetext{
${ }^{10}$ T. d. a.: "En los seis primeros episodios de su primera temporada, Perdidos desarrolla treinta y tres arcos, mientras que el episodio cuarto comprende nueve de ellos. Solo dos de dichos arcos son introducidos y cerrados en ese episodio [...] seriales como Cómo conocí a vuestra madre (USA 2005-, CBS) también exhiben elementos entretejidos — aunque emerjan más desde detalles que desde arcos enteros (como por ejemplo, el paraguas amarillo de la madre de los niños, que esmencionado una y otra vez como un hilo conductor de la historia). Y por si no fuera suficiente: la complejidad narrativa está constituida por el hecho de que algunas conexiones narrativas son mostradas en el primer episodio y no son finalizadas hasta el último episodio de la misma temporada. Los arcos no solo se saltan una temporada, sino varias".
} 
en lo ocurrido en episodios previos para desentrañar el nudo de líneas argumentales, nos interesa acercarnos a otro punto de interés narratológico: los juegos con las lagunas informativas. Como expone el crítico cultural Steven Johnson (2005: 63-64):

Narratives that require that their viewers fill in crucial elements take that complexity to a more demanding level. To follow the narrative, you aren't just asked to remember. You're asked to analyze. This is the difference between intelligent shows, and shows that force you to be intelligent ${ }^{11}$.

En estos "programas que te fuerzan a ser inteligente" existe un grado superior de complejidad sobre el de los seriales complejos descritos por Piepiorka, uno que trasciende el esquema multitrama y le añade ambigüedad a la manera de las series dramáticas que conforman nuestra muestra de análisis: Hannibal (Fuller, NBC: 2013-2015), The Affair (Treem y Levi, Showtime: 2014-) y Twin Peaks (Lynch y Frost, ABC y Showtime: 19901991 y 2017).

\section{METODOLOGÍA}

Partimos de las proyecciones sobre las maneras de narrar trazadas en otros trabajos sobre la poética histórica del cine de dos de los modos de narración planteados por David Bordwell (1996): el clásico y el de arte y ensayo. Bordwell emplea tres categorías a la hora de caracterizar las estrategias de transmisión de información de la narración con el fin de acometer con propiedad el estudio de "la forma en que el estilo del filme y la construcción del argumento manipulan el tiempo, el espacio y la lógica

\footnotetext{
${ }^{11}$ T. d. a.: "Las narrativas que requieren que sus espectadores rellenen elementos cruciales llevan esa complejidad a un nivel de exigencia superior. Para seguir una narrativa no solo se espera que recuerdes. Se espera que analices. Esta es la diferencia entre programas inteligentes y programas que te fuerzan a ser inteligente".
} 
narrativa para permitir al espectador construir un desarrollo específico de la historia" (1996: 61).

La más importante de las tres es la comunicabilidad (communicativeness). Bordwell se refiere a la comunicabilidad como el grado de efectiva comunicación de los saberes al espectador que la narración se permite en función de una estimación de su máximo posible. Por la potencial polisemia del término, conviene explicitar las consideraciones que Emilio Garroni aporta sobre el concepto de comunicabilidad aplicado desde la semiótica (1973: 27-32). Para Garroni la comunicabilidad de un fenómeno artístico es una condición formal necesaria por esquiva y obscura que sea su estrategia comunicativa (caso de las vanguardias artísticas): “[o] hay fenómeno de comunicación o no lo hay: si lo hay, aunque sea en forma extremadamente reducida, desde luego es comunicable". De aquí deriva la afirmación de que "la comunicabilidad (en distintos grados) no es ni debe ser un criterio rudimentario de valoración de los productos artísticos en cuestión”, sino que, más bien, “en el aspecto analítico e historiográfico, simplemente se trata de un dato objetivo y de un condicionamiento histórico, que el estudioso deberá tener en cuenta para mejor comprender". De esta manera, Garroni distingue dos niveles de comunicabilidad. Por un lado, la ya citada "comunicabilidad formal" (inherente a cualquier acto comunicativo). Por otro lado, la "comunicabilidad material", que alude a que para la comprensión óptima del mensaje de un acto comunicativo se requiere de una especificidad de saberes concretos, muchas veces únicamente compartidos por un grupo reducido y aislado de la gran masa de los comunicantes. En nuestro caso, el estudio de la comunicabilidad propuesta por Bordwell se centra en la "comunicabilidad material" de las sucesivas poéticas de los distintos modos de narración. En concreto, Bordwell propone una gradación no valorativa de la comunicabilidad en la narración fílmica para identificar con propiedad los parámetros textuales de maneras de hacer cine que se alzan como desviaciones del modelo clásico. Es decir, empleando referentes que ambos manejan en sus textos, Bordwell trata de definir a través del cine de Godard o de Antonioni lo que Garroni identifica 
en ellos como "una poética de la comunicación perturbada".

El máximo de comunicabilidad queda establecido, según Bordwell, en buena medida por las gradaciones de las otras dos categorías. La primera es la cognoscibilidad, que depende a su vez de dos factores. El primero de ellos es el grado de restricción. ¿Se limita el conocimiento de la narración a los saberes de un personaje concreto, como el caso de Jeff en La ventana indiscreta (Rear Window, Hitchcock, 1954)? La restricción presenta entonces un grado alto. ¿O, por el contrario, la narración ofrece más información de la que un solo personaje de la diégesis puede abarcar? Si así es estamos ante un bajo grado de restricción, común entre filmes con protagonismo coral. El segundo de los factores es el grado de profundidad de ese conocimiento, que guarda relación directa con la subjetividad de saberes: a mayor profundidad, mayor subjetividad. De esta manera, estadios bajos de profundidad nos remiten a lo que ve y/u oye un personaje (siguiendo con La ventana indiscreta, la ocularización en Jeff mirando a través de sus prismáticos), mientras que una alta profundidad permite a los espectadores ver procesos mentales subjetivos como ensoñaciones y delirios.

La otra categoría es la autoconsciencia, la mostración de un reconocimiento por parte de la narración de estar interpelando a un público. Marcas enunciativas, como las panorámicas voyeristas por los ventanales del patio de vecinos de La ventana indiscreta, de clara función expositiva, son señales evidentes de autoconsciencia. La gradación de la autoconsciencia de este tipo de marcas — entre las que también se incluyen las repeticiones, las rupturas de la cuarta pared o las voces over- depende en buena medida de cómo la revelación de la existencia de la narración al público condiciona el argumento dentro de las convenciones genéricas y de los modos de producción. Por ejemplo, la gran autoconsciencia de las interpelaciones a la cámara es relativa en los musicales por ser una convención genérica, mientras que, pese a su autoconsciencia, los primeros planos de las estrellas son una convención del cine de los estudios del Hollywood clásico. 
A modo de recapitulación, la narración restrictiva de La ventana indiscreta es, sin embargo, notablemente comunicativa, ya que durante el grueso del metraje nos permite acceder a los saberes de Jeff (alta profundidad), salvo en aquellos momentos en los que, rompiendo con las normas que la película introduce con respecto a las anteriores categorías, explicita las lagunas de conocimiento de Jeff, a su vez ligadas a la adscripción del filme al género detectivesco: "la narración puede contar más, pero no lo hace" (1996: 59), un signo de autoconsciencia moderada. Como se ha demostrado, la comunicabilidad es una categoría especialmente útil tanto para explicitar las lagunas de la narración, consecuencia de una desviación de la norma interna de comunicabilidad de un filme, como para sopesar las motivaciones transtextuales, como el género (suspense en el policíaco, sorpresa en el terror), respecto a las demandas estructurales intrínsecas. Aunque, como el propio Bordwell advierte, "[e]n general, las películas narrativas modulan constantemente la amplitud y profundidad del conocimiento de la narración" (1996: 58), poner la atención en el desarrollo de estas modulaciones, en especial en las fases más complejas de un filme, ayuda a arrojar luz sobre la ambigüedad de la etiqueta de complejidad narrativa.

Con estas categorías, Bordwell fija los parámetros de dos modos que entiende opuestos: la narración clásica y la narración de arte y ensayo. El modo clásico queda determinado por una baja restrictividad y una alta profundidad (la narración hace incursión en la subjetividad de un amplio número de personajes), una moderada autoconsciencia (pese a no hacer interpelaciones directas a los espectadores sí son notables las marcas enunciativas como música extradiegética o títulos de crédito) y una comunicabilidad moderada (la narración sólo guarda para sí los hechos por venir en el tiempo diegético). En analogía al modo de narración clásico cinematográfico, la narración televisiva convencional presenta unos patrones similares en lo referente a las tres categorías enunciadas por Bordwell. El reparto coral de sitcoms y telenovelas motiva una baja restrictividad, que en el caso de estas últimas redunda, por su característica afectación 
melodramática, en una potencialmente alta profundidad en la psiquis de los personajes. Pese a que los eventos diegéticos no incurran en ello, la ficción televisiva obtiene un grado moderado de autoconsciencia por las interrupciones inherentes a su integración en la parrilla televisiva que, en el caso de las televisiones comerciales, están además atravesadas por bloques publicitarios. Finalmente, a efectos de comunicabilidad los valores son similares, y prueba de ello es la popularidad del género policiaco (que añade a su normativa ocultación selectiva de la información la variable de la duración propia del melodrama serial) a lo largo de la historia de la televisión.

Por su parte, el modo de narración de arte y ensayo presenta una baja comunicabilidad resultado de una alta restrictividad y profundidad en la psiquis de los protagonistas y una autoconsciencia irónica, consciente no sólo de condición de relato sino de su propia ambigüedad (y de la crítica subyacente que ello supone sobre los postulados del clasicismo narrativo).

Recogiendo la metodología bordwelliana, Eleftheria Thanouli (2009) propone la existencia de un nuevo modo de narración que abarca un abanico de opciones estilísticas muy específicas 12 y que son empleadas en el cine desde la década de 1970. Thanouli articula lo que identifica como un nuevo modelo de hacer cine a través de una poética histórica del cine postclásico. Su estudio arroja una serie de normas concretas de este nuevo paradigma de la narración fílmica frente al modelo clásico:

L La narración postclásica presenta un alto grado de autoconsciencia en la exhibición de un grado explícitamente alto de cognoscibilidad (baja restrictividad, alta profundidad) sobre los eventos de la historia.

$\checkmark$ A diferencia de la narración clásica, cuya omnisciencia permanece restringida salvo por las marcas narrativas durante las secuencias de apertura y cierre, el paradigma postclásico revela

\footnotetext{
${ }^{12}$ Algunas de ellas desarrolladas en profundidad en Palao-Errando, Loriguillo-López y Sorolla-Romero (2018).
} 
constantemente su condición de relato mediante la transmisión de información a través de canales que transcienden tanto la subjetividad de los personajes como los diálogos entre ellos (fuente predilecta de la información en el modelo clásico). Paradigmática de esta autoconsciencia es la narración hiperdetallística en Amélie (Jeunet, 2001) o las secuencias de montaje de El club de la lucha (Fight Club, Fincher, 1999).

$\checkmark$ Pese al alto grado de autoconsciente omnisciencia, la comunicabilidad en el cine postclásico se mantiene en un nivel alto con el fin de ofrecer una progresión narrativa de principio a final que no deje lagunas ni cabos sueltos en su argumento.

\begin{tabular}{c|cccc}
\hline & \multicolumn{2}{c}{ Cognoscibilidad } & Autoconsciencia & Comunicabilidad \\
& Restrictividad & Profundidad & & \\
\hline Clásico & Baja & Alta & Baja-moderada & Moderada \\
Arte y ensayo & Alta & Alta & Constantemente alta & Baja \\
Postclásico & Baja & Alta & Explícitamente alta & Alta
\end{tabular}

Tabla 2. Resumen de las gradaciones de las categorías de estrategias narrativas para los modos de narración clásico, de arte y ensayo (Bordwell, 1996) y postclásico (Thanouli, 2009). Fuente: elaboración propia.

\section{CASOS DE ESTUDIO}

\subsection{La cognoscibilidad en el detective contemporáneo: Hannibal}

Como en las "puzzle films", la emergencia de los narradores poco fiables merece atención por su ubicuidad en buena parte de los títulos adscritos a la televisión compleja. Es de nuevo a través de una focalización interna profunda en estos protagonistas, aquejados de los más variados trastornos mentales, sobre los que la narración (altamente restrictiva) se 
apoya a la hora de divulgar información diegética. En este caso, nos aproximamos a la narración compleja en la ficción policiaca, imbuida plenamente en una recurrente "estética de la vigilancia" desde el 11S (Cascajosa Virino, 2018: 147), y que presenta ejemplos notables por la reincidencia en el tropo del investigador trastornado que, en última instancia, pone sobre la mesa el conflicto entre la normalización e integración de los enfermos mentales en la sociedad y la recurrente locución de Juvenal "Quis custodiet ipsos custodes?"13.

Hannibal es un caso paradigmático del drama policiaco en la era de la complejidad no sólo por tener un narrador poco fiable en Will Graham (Hugh Dancy), sino por ser un ejemplo de cómo construir un intricado juego sobre la cognoscibilidad de la narración desde una de las franquicias más influyentes del audiovisual popular. Este Graham sigue siendo el criminólogo estrella del FBI pero aquí es representado como un frágil y ensimismado individuo cuya percepción está atravesada por una imaginación desbordante que le lleva a empatizar de tal manera con los asesinos a los que trata de entender que recrea en su cabeza los cruentos crímenes que investiga. Sus escabrosas revisitaciones de las escenas de los crímenes no sólo transmiten a los espectadores la creciente fragilidad psicológica de Graham tras cada investigación (quedando sus valores

\footnotetext{
${ }^{13} \mathrm{El}$ tropo del investigador perturbado tiene recorrido en otras dos de las más estimulantes series policiacas de los últimos años. En River (Morgan, BBC One: 2015), el inspector John River (Stellan Skarsgård) interactúa con personas fallecidas que han marcado su pasado. La última incorporación a sus imaginarios contertulios es la sargento Stevie Johnson (Nicola Walker), su compañera de patrulla, cuyo asesinato se propone resolver. En Marcella (Rosenfeldt, ITV: 2016), la sargento Marcella Backland (Anna Friel), no recuerda si ha tenido algo que ver en la muerte de la amante de su marido. Su maña investigadora a la hora de realizar averiguaciones clave para la resolución de una serie de asesinatos justifica el encubrimiento que compañeros ofrecen a Marcella por su condición: sufre de apagones de conciencia tras los que no recuerda qué ha hecho. La narración, manteniendo una estricta restricción en su parte consciente como focalizadora del relato, mantiene esta laguna central sin resolver a la conclusión de la temporada, ya que en ningún momento desvela explícitamente la naturaleza de su implicación en ninguna muerte. Son sólo las pistas que la propia Marcella trata de ocultar tras despertar de sus desvanecimientos las que apuntan a su presumible tendencia asesina.
} 
como policía en entredicho, en línea con lo que señalaba Rimmon-Kenan), sino que constituyen unas secuencias de alta densidad en cuanto a los niveles de la narración. En estos ejercicios de abstracción, la focalización interna en Graham se focaliza, a su vez, en la percepción y motivación de los homicidas. Estas analepsis imposibles en términos de coherencia están precedidas por rebobinados y times lapses invertidos (marcas enunciativas de alta autoconsciencia) y son indefectiblemente cerradas por las palabras de un Graham catatónico tras el trance identitario: "This is my design"14.

Tras sentir placer al abatir al verdugo de Minnesota, Graham inicia voluntariamente terapia con el Dr. Hannibal Lecter (Mads Mikkelsen), del que de forma inexplicable sospecha y, a la vez, por el que se siente atraído. La subsiguiente exposición frecuente a la manipulación psicológica y juegos mentales de Lecter empeoran su condición hasta el punto de ser internado en un pabellón psiquiátrico. Como expresa significativamente el propio Graham para mayor autoconsciencia, "I have lost the plot. I am the unreliable narrator of my own story" 15 en \#02x02: Sakizuke, Hunter, NBC: 2014. Además de condicionar la disposición de la información a través de la syuzhet mediante los flashbacks de recreaciones explícitas, la función focalizadora del hamletiano Graham (García Martínez, 2018: 100) sirve para representar la escalada de perturbación continua en los siguientes episodios, en los que es testigo de eventos imposibles. En \#02x03: Hassun, Medak, NBC: 2014, Graham se ejecuta a sí mismo en la silla eléctrica en una pesadilla justo antes del juicio que tiene que dictaminar su presunta implicación en los asesinatos. En la posterior vista, Lecter sube a declarar ante el juez y, a los ojos de Graham toma la forma de un wendigo, un espíritu caníbal en la mitología algonquina. Ambos casos son representativos de la turbación de la narración, que en adelante parecerá contagiada por la condición de Graham en su tortuoso día a día, plagado de ensoñaciones potencialmente premonitorias y visiones terroríficas difíciles tan difíciles de disociar de la estilizada diégesis (Piñeiro Otero, 2016; Medina Contre-

\footnotetext{
14“"Ese es mi propósito" en la versión española.

${ }^{15}$ T. d. a.: "He perdido el argumento. Soy el narrador poco fiable de mi propia historia".
} 
ras, 2018: 202) como de poder aprehender la historia desde los esquemas de la coherencia y de la lógica causal.

\subsection{La autoconsciencia delatora de faltas: The Affair}

Como observamos en el caso anterior, el cambio la narración tiene también que ver con la transición del paradigma del héroe de acción sin mácula al protagonista típicamente complejo contemporáneo, “[s]carred and wounded, heroic nonetheless" (Shimpach, 2010: 189). La revalorización del antihéroe tras los Los Soprano (The Sopranos, Chase, HBO: 1999-2007) parece influir en su consolidación como arquetipo de protagonista focalizador con problemas éticos y morales que genera simpatías entre los espectadores pese a ser un mafioso, un narcotraficante o un adultero reincidente (Canet y García-Martínez, 2018).

Este último es el caso de Noah Solloway (Dominic West), uno de los focalizadores de The Affair. La fiabilidad de cada uno de los personajes-narradores de la serie se pone a prueba a través de un mecanismo narrativo reminiscente a Rashomon (Kurosawa, 1950): el contraste de subjetividad, recurso plenamente autoconsciente de la narración, que yuxtapone mediante repetición un tiempo diegético desde el punto de vista de dos personajes distintos por episodio. De esta manera, el piloto - \#01x01: 1, Mylod, Showtime: 2014 - está escindido en dos partes. En la primera, titulada "Noah" se nos ofrece la versión de Solloway, un escritor neoyorkino acomodado, casado y padre de tres hijos, de su aventura con Allison Bailey (Ruth Wilson), una camarera del pueblo en el que veranea. La segunda parte, titulada “Allison”, está focalizada en Bailey, una joven madre que sufre las secuelas psicológicas de la reciente muerte de su hijo. Mediante el contraste de las versiones no sólo se complementan las motivaciones de los personajes no accesibles desde la otra parte (alta restrictividad), sino que los espectadores comienzan a conocer que los impulsos y deseos de los focalizadores modifican su relato hasta que las versiones son, en muchos casos opuestas. Por ejemplo, en el primer encuentro de 
Solloway y Bailey, el cabello y la longitud de la falda de ella varían según el focalizador: en la versión de él, lo lleva suelto y viste una falta corta; mientras que en la de ella, lo lleva recogido y viste una más larga. Se trata de marcas enunciativas que advierten de la naturaleza promiscua de Noah, de nuevo un reprobable y poco fiable focalizador. Sin embargo, el resto de focalizadores — desde la propia Bailey (en tratamiento psicológico tras la muerte de su hijo pequeño), hasta sus respectivos cónyuges y amantes en las temporadas siguientes - se muestran como mentirosos e interesados en el mejor de los casos. La eminencia de Noah como protagonista dominante parece evidenciarse cuando, en un giro hacia el thriller psicológico en la tercera temporada, sufre de visiones persecutorias fruto de estrés tras varios eventos traumáticos estar en prisión por un asesinato que no ha cometido, asimilar sus dos divorcios y la muerte de su padre. Las continuas idas y venidas temporales para abarcar dichos momentos en la diégesis - analepsis homodiegéticas incompletas (únicamente resueltas en la conclusión de la temporada) de incluso décadas atrás del presente diegéticos - evidencian la complejidad narrativa en la que incurre la serie.

De esta manera, el rasgo queda explicitado mediante dos marcas enunciativas evidentes: los juegos con la cronología (en especial con la poco habitual categoría de la frecuencia: vemos en muchos episodios repetidos los mismos eventos diegéticos desde dos puntos de vista) y los intertítulos que dividen indefectiblemente cada episodio por la mitad y que anuncian quién va a ser el focalizador del fragmento. Así, la complejidad narrativa opera en pos del discurso subyacente: la realidad es discutible y está a prueba de coherencia. Especialmente, en el choque de versiones que los actos de violencia machista suscitan entre las partes implicadas. Esta ambigüedad narrativa en pos de la progresiva sensibilización social a través de la complejidad en la narración es recogida también por series de reciente producción como Liar (Williams y Williams, ITV: 2017) o Alias Grace (Harron, CBC: 2017) con un nivel de ambigüedad discursiva menor. 


\subsection{La comunicabilidad opaca: Twin Peaks}

Los límites de la comunicabilidad audiovisual de la narración en la ficción televisiva comercial quedan definidos por Twin Peaks. Como sentencia Elsaesser en referencia a su carrera cinematográfica, parece que la obra de Lynch, independientemente de su formato, "siempre encuentra una nueva forma de conmocionar, enfrentar unos a otros y distribuir de forma asimétrica causas y consecuencia, actor e intenciones, recuerdos y previsiones" (2013: 18). Mucho se ha escrito desde la prolífica aproximación académica a Twin Peaks, que ha repuntado en los últimos años merced al estreno de la tercera temporada de la serie —el "cliffhanger más largo de la historia de la televisión" (Ros, 2017)—. Pese a que su narración parece en origen ceñirse a un whodunit serial de motivación realista — “¿Quién ha matado a Laura Palmer?"-, el extrañamiento que emerge de las pesadillas del focalizador principal, el agente especial del FBI Dale Cooper (Kyle MacLachlan), así como las revelaciones oníricas en la Habitación Roja (Skoptsov, 2015), devienen tanto en escaramuzas con entidades malignas como en encuentros proféticos con la propia Laura (Sheryl Lee) y otros personajes en las Logias Negra y Blanca. Estos espacios se unen en la diégesis mediante la investigación del equipo "Blue Rose" del FBI: los encuentros con habitantes de otras dimensiones alumbrados por el ente madre, Judy. Una de estas entidades, BOB (Frank Silva), es el espíritu maligno que posee tanto al padre y asesino de Laura Palmer (Ray Wise) como al doppelgänger maligno de Cooper, que confina a su original en estos espacios y lo suplanta en la realidad diegética.

A diferencia de las dos primeras temporadas — con rasgos propios de complejidad por su eclecticismo genérico y el surrealismo emergente (Creeber, 2004: 49)—, la tercera temporada comienza con una explosión de líneas argumentales secundarias alrededor de un creciente números de personajes y de visiones presuntamente alegóricas que, aunque luego guarden relación entre sí a la manera de los seriales complejos multitrama, también dejan cabos sueltos sin solución de continuidad: ¿qué le ha 
sucedido a Audrey (Sherilyn Fen) tras su chocante despertar ante un espejo en \#03x16: Part 16, Lynch, Showtime: 2017? ¿Qué ha sido de Sarah Palmer (Grace Zabriskie) tras destrozar el retrato de su hija en el episodio final? ¿Están Cooper y Carrie Page (trasunto de doppelgänger de Laura) en una realidad alternativa, en el pasado o en el futuro? La comunicabilidad de la narración con respecto a toda esta serie de cuestiones es mínima, superando en oscuridad narrativa a la de la televisión compleja que hemos analizado.

En paralelo a estas lagunas de información permanentes, las representaciones abstractas del celebrado $\# 03 \times 08$ : Part 8, Lynch, Showtime: 2017, intercaladas por una puesta en escena antinatural y recursos expresivos altamente manieristas — desenfoques, stop-motion, blanco y negro - denotan una voluntad de entender el audiovisual comercial como no estrictamente narrativo. Se trata de una intencionalidad consecuente si se atiende a la cognoscibilidad de la narración, generalmente no restrictiva y profunda, mediante la cual accedemos a fenómenos sobrenaturales crípticos. La autoconsciencia en Twin Peaks es explícitamente alta desde varios niveles: desde la remediación de fragmentos de las anteriores temporadas y del filme precuela Twin Peaks: Fuego camina conmigo (Twin Peaks: Fire Walk with Me, Lynch, 1992) — justificados mediante flashbacks_-, a la relación actor-actante (justificada por la diégesis por la existencia de los doppelgänger y “tulpas”), pasando por la convención caduca de la actuación musical diegética durante la mayoría de los episodios en el Roadhouse de Twin Peaks. Esta autoconsciencia no está exenta de homenajes al cine. En \#03x15: Part 15, Lynch, Showtime: 2017 es el visionado de El crepúsculo de los dioses (Sunset Boulevard, Wilder, 1950) —en concreto cuando Cecil B. DeMille menciona a un tal "Gordon Cole", tocayo del jefe y amigo de Cooper - el que hace salir a Cooper del estado catatónico en el que está desde su vuelta al mundo diegético real en el cuerpo de Dougie Jones. Finalmente, tras dar señales de vida por fin al FBI, el propio Cole (David Lynch) se muestra perplejo por la aparición de Cooper bajo la identidad de Jones, explicitando en voz alta lo que 
los espectadores llevan preguntándose desde el principio de la serie: ¿es Cooper "Dougie"? La secuencia es especialmente autoconsciente porque es el propio Lynch el que interpreta a Cole.

\section{DISCUSIÓN}

Pese a que nuestra metodología de análisis está enfocada en la particularidad de lo que presentamos como textos televisivos complejos en lo relativo a la comunicabilidad, la industria televisiva (como toda industria cultural) se ciñe a unos patrones - de duración, de géneros y de pactos tácticos con los espectadores - para reducir en lo posible los riesgos derivados de la producción de ficción. De esta manera, desde productoras y distribuidoras se enfrentan a un contexto competitivo en el que se reinventan continuamente fórmulas creativas que destaquen de entre la sobresaturada oferta pero, al mismo tiempo, desde una conservadora inquietud en lo experimental de sus apuestas si éstas son susceptibles de chocar con la familiaridad de los espectadores (siendo el tándem compuesto por share y por los anunciantes el principal culpable de la repetida crítica a lo formulaico de la ficción televisiva). En este sentido, la ambigüedad narrativa de la televisión compleja está de nuevo condicionada por su continuidad deseada por los showrunners para el desarrollo del relato. Pocas son las series conclusas de entre las propuestas que incurren en una flagrante oscuridad narrativa como consecuencia de una baja comunicabilidad. Aunque polémico por evidenciar que su prometedora ambigüedad no conducía a ninguna parte, el cierre de Perdidos supone un manifiesto intento de llegar a una clausura, algo que otras series no pudieron ni siquiera efectuar al ser canceladas con anterioridad — caso de FlashForward (Braga y Goyer, ABC: 2009-2010)_. De la misma manera, pese a su manierismo narrativo, tanto rara avis como El detective cantante (Potter, BBC1: 1986) como incursiones en la ciencia-ficción como Fringe: Al límite (Abrams, Kurtzman y Orci, Fox: 2008-2013) y The Leftovers (Lindelof y Perrota, HBO: 2014-2017) concluyen incluso con 
la reconciliación heterosexual, epílogo por antonomasia de la narración clásica MRI (Gómez Tarín, 2018: 49-50). De esta manera, consideramos que el modo de narración de la televisión compleja presenta valores análogos a los del modo de narración del cine postclásico, mucho más comunicativo que la del modo de narración de arte y ensayo (Tabla 3).

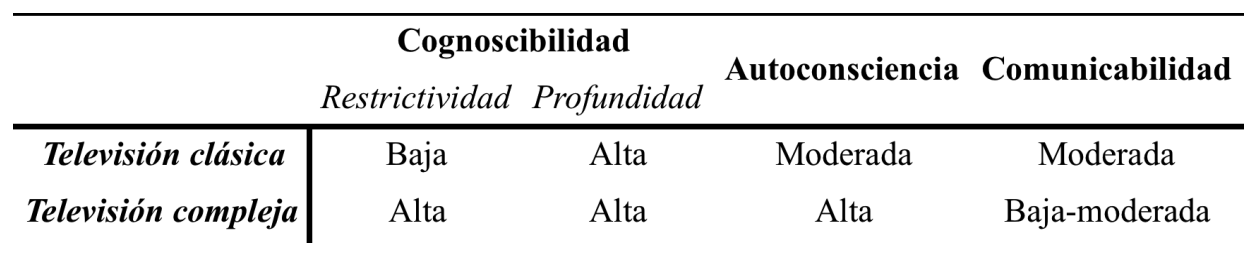

Tabla 3. Propuesta de gradación de las categorías de estrategias narrativas del modo de narración de la televisión compleja. Fuente: elaboración propia.

Los hallazgos de nuestro análisis narratológico aplicado a nuestra muestra apuntan a que el modo de narración de la televisión compleja cumple generalmente con una cognoscibilidad altamente restringida en unos protagonistas focalizadores cuya subjetividad — condicionada normalmente por problemas mentales - es ofrecida merced a una alta profundidad. La narración es también altamente autoconsciente, algo justificado no sólo por el uso de toda una batería de recursos del audiovisual postclásico — mise en abîme, homenajes, citas, parodia y pastiche-, sino también por la naturalización de las interrupciones (optimización de los cliffhangers) y limitaciones episódicas (previously, secuencias de apertura, preending y ending; Bort Gual, 2012: 241) del flujo televisivo. Su similitud con el modo de narración de las películas no solo es prueba de la permeabilidad entre ambos medios en lo referente a la ficción, sino que también apunta a la progresiva disolución de los rasgos narrativos que los han dividido históricamente en un contexto de crisis de la distribución convencional y de emergencia de plataformas VoD (como Netflix, Amazon Studios o Hulu) devenidas en centros de producción multipantalla.

En relación a estas consideraciones debemos señalar las limitaciones 
de este estudio. La construcción de una poética de la televisión compleja a la que apuntamos debe acabar de concretarse atendiendo al contexto. Resulta indispensable en este sentido explicitar de manera sólida la naturaleza de la relación entre la complejidad de la narración en estos filmes y de la convulsa situación de la industria que las produce. De entre diversos elementos de interés para evaluar el grado de potencial experimentación - como la atención a las maneras de producir y de presupuestar en las cadenas televisivas - se antoja esencial atender a la distribución cualitativa y cuantitativa de las series complejas. El potencial estado de culto que adquieren muchas de ellas en el mercado de las ediciones domésticas - un factor dependiente en la actualidad de su inclusión en los citados catálogos de plataformas de VoD - aparece como una variable de gran importancia. Exploraciones previas sobre el alcance del circuito del arte y ensayo internacional (Iordanova, 2008 y Lobato, 2012) o sobre la consolidación del cine independiente en Estados Unidos (King, Molloy y Tzioumakis, 2013) apuntan hacia una posible vía desde la que partir.

\section{CONCLUSIONES}

$[\mathrm{T}]$ hese were storytelling devices that you would have found only in avant-garde narrative thirty or forty years ago: in Pinter, or Alain Robbe-Grillet, or Godard. You might have been able to fill a small theater in Greenwich Village with an audience willing to parse all that complexity in 1960, but only if the Times had given the play a good review that week. Forty years later, NBC puts the same twisted narrative structure on prime-time television, and 15 million people lap it up ${ }^{16}$ (Johnson, 2005: 89).

\footnotetext{
${ }^{16}$ T. d. a.: "Estos eran mecanismos narrativos que habrías encontrado únicamente en la vanguardia de hace treinta o cuarenta años: en Pinter, Alain Robbe-Grillet o Godard. En los sesenta podías haber llenado una pequeña sala en Greenwich Village con un público dispuesto a lidiar toda esa complejidad, pero solo si el Times hubiera dado esa semana una buena reseña a la representación. Cuarenta años más tarde, la NBC pone esa misma estructura narrativa retorcida en prime time y quince millones de personas la acogen con
} 
La televisión compleja está revolucionando la experiencia audiovisual mediante un barniz social. Las heterogéneas prácticas paratextuales en torno a las series complejas hacen complicado asentar una definición inequívoca de lo que es una serie de culto, más si la televisión de culto es tan aprehensible desde una perspectiva textualista como desde las relaciones entre industria y públicos (Pearson, 2010: 16). De entre estas últimas destacan las prácticas entre pares que los espectadores realizan para intercambiar aproximaciones hermenéuticas (más o menos fantasiosas) sobre las lagunas y ambigüedades de las series. La intensidad de la actividad tradicionalmente asociada al espectador catódico se ha expandido fuera de sus fronteras convencionales, regidas primordialmente por su emisión, y ahora es posible seguir aprehendiendo los cabos sueltos y disonancias narrativas intencionadas a través de múltiples plataformas de manera independiente. Numerosos televidentes trascienden su (supuesta) pasividad y se lanzan a la elaboración de planos, wikis y comunidades virtuales interpelados por la complejidad serial televisiva. Este tipo de interacciones revelan en última instancia cómo se integra la variante de la complejidad narrativa dentro de las formas de consumo de las series televisivas contemporáneas. Como indica Mittell: "[i]t's not just that audiences are active, but that texts are explicitly activating, designed to stimulate viewers, strategically confuse them, and force them to orient"17 (2013: 178). El retorno económico tangible generado tanto por la redifusión como por el alquiler y las ventas de ediciones domésticas - trufadas muchas veces de extras, comentarios del director y behind the scenes que, en la búsqueda espectatorial de sentido se alzan como faros de conocimiento entre la oscuridad narrativaconstituyen argumentos de rentabilidad para producir televisión compleja. La domesticación del flow televisivo (Williams, 1974) en formato físico permite incurrir en prácticas como el coleccionismo y el denominado sig-

entusiasmo".

${ }^{17}$ T. d. a: "no se trata únicamente de que las audiencias sean activas, sino de que los textos están orientados explícitamente hacia la activación, diseñados para estimular, confundir estrategicamente y forzar a los espectadores a orientarse". 
nificativamente "forensic fandom", manifestaciones explícitas, en definitiva, de lo que los manuales de narrativa indican sobre las tensiones de la homología: "[p]ueden contribuir a una lectura más intensa, tan intrincadas que exijan el mayor de los esfuerzos para seguir la historia" (1985: 60).

De esta manera, el potencial persuasivo de las series de ficción compleja en esta era en la que se encapsulan emisiones para su distribución material se convierte en uno de los activos que avalan títulos que, aunque arranquen de manera parca en cuanto a espectadores, recuperan e incluso generan beneficios a largo plazo en el mercado doméstico. Otras ventanas (sean o no sancionadas por los propios productores) emergen como improvisadas muletas de conocimiento para que otros espectadores se ubiquen (cronológica y espacialmente) en una narración que, salvo el caso excepcional de Perdidos, pone en dificultades hermenéuticas a los espectadores reacios a alejarse a los convencionalismos seriales. El enganchar de manera multidimensional a los públicos, uno de los rasgos de las producciones culturales comerciales del s. XXI, tiene un gran potencial en el caso de la televisión compleja.

\section{REFERENCIAS BIBLIOGRÁFICAS}

ABRAMS, J. J. y LINDELOF, D. (Productor) y LIEBER, J.; ABRAMS, J. J. y LINDELOF, D. (Creador). (2004-2010). Perdidos [Serie de televisión]. Oahu: ABC.

ABRAMS, J. J.; BURK, B.; KURTZMAN, A. y ORCI, R. (Productor) y ABRAMS, J. J.; BURK. B.; KURTZMAN, A. y ORCI, R. (Creador). (2008-2013). Fringe: Al límite [Serie de televisión]. Vancouver: Fox.

ARISTÓTELES (2004). Poética. Madrid: Alianza.

ATWOOD, M. (Productor) y HARRON, D. (Creador). (2017). Alias Grace [Serie de televisión]. Canadá y Estados Unidos: CBC.

BAL, M. (1985). Teoría de la narrativa (una introducción a la narratolo- 
gía). Madrid: Cátedra.

BIGNELL, J. (2007). "Seeing and Knowing: Reflexivity and Quality". En Quality TV. Contemporary American Television and Beyond, J. McCabe y K. Akass (eds.), 158-170. New York: I.B. Tauris.

BORDWELL, D. (1996). La narración en el cine de ficción. Barcelona: Paidós.

(2002). "Film Futures". SubStance 31.1, 88-104.

BORT GUAL, I. (2012). Nuevos paradigmas en los telones del relato audiovisual contemporáneo: partículas narrativas de apertura y cierre en las series de televisión dramáticas norteamericanas. Tesis doctoral. Universitat Jaume I.

BRACKETT, C. (Productor) y WILDER, B. (Director). (1950). El crepúsculo de los dioses [Film]. Estados Unidos: Paramount Pictures.

BRAGA, B. y GOYER,D. S. (Productor) y BRAGA, B. y GOYER D. S. (Creador). (2009-2010). Flashforward [Serie de televisión]. Los Ángeles: ABC.

BRANIGAN, E. (2002). "Nearly True: Forking Plots, Forking Interpretations: A Response to David Bordwell's Film Futures". SubStance 31.1, 105-114.

BUCKLAND, W. (2009). "Introduction: Puzzle Plots". En Puzzle films: complex storytelling in contemporary cinema, W. Buckland (ed.), 1-12. Malden: Wiley-Blackwell.

(2014). "Introduction: Ambiguity, Ontological Pluralism, and Cognitive Dissonance in the Hollywood Puzzle Film". En Hollywood Puzzle Films, W. Buckland (ed.), 1-14. London: Routledge.

BURCH, N. (1987). El tragaluz del infinito: contribución a la genealogía del lenguaje cinematográfico. Madrid: Cátedra.

CAMERON, A. (2008). Modular narratives in contemporary cinema. New York: Palgrave Macmillan.

CANET, F. y GARCÍA MARTÍNEZ, A. N. (2018). "Respuestas ambivalentes ante la moralidad ambigua del antihéroe: Tony Soprano y Water White como casos de estudio". Palabra Clave 
21.2, 364-386.

CASCAJOSA VIRINO, C. (2005). Prime time: las mejores series de TV americanas, de C.S.I. a Los Soprano. Madrid: Calamar.

(2018). "The Night Of: una estética de la vigilancia y la justicia para los Estados Unidos del pos-11S". En La estética televisiva en las series contemporáneas, M. Huerta Floriano y P. Sangro Colón (eds.), 143-161. Valencia: Tirant Humanidades.

CHASE, D.; GREY, B. y GREEN, R. (Productor, y Chase, D. (Creador). (1999-2007). Los Soprano [Serie de televisión]. Nueva Jersey: HBO.

CREEBER, G. (2004). Serial television: big drama on the small screen. London: British Film Institute.

(2013). Small screen aesthetics: from TV to the Internet. Basingstoke: Palgrave Macmillan.

DESCHAMPS, J. P. (Productor) y JEUNET, J. P. (Director). (2001). Amélie [Film]. Francia: Canal +, France 3 Cinéma, UGC.

EIG, J. (2003). "A beautiful mind(fuck): Hollywood structures of identity". Jump Cut: A Review of Contemporary Media 46 (también en https://www.ejumpcut.org/archive/jc46.2003/eig.mindfilms/ [20/04/2018]).

ELSAESSER, T. (2009). "The Mind-Game Film". En Puzzle films: complex storytelling in contemporary cinema, $\mathrm{W}$. Buckland (ed.), 1341. Malden: Wiley-Blackwell.

(2013). "Los actos tienen consecuencias. Lógicas del mind-game film en la trilogía de Los Ángeles de David Lynch". L'Atalante. Revista de estudios cinematográficos 15, 7-18.

FEATHERSTONE, J.; LEVIN, M.; MORGAN, A. y RICHER, L. (Productor) y MORGAN, A. (Creador). (2015). River [Serie de televisión]. Londres: BBC.

FIENBERG, G. (Producer) y LYNCH, D. (Director). (1992). Twin Peaks: Fuego camina conmigo [Film]. Estados Unidos: CIBY Pictures.

FROST, M. y LYNCH, D. (Productor) y FROST, M. y LYNCH, D. 
(Creador). (1990-1991, 2017). Twin Peaks [Serie de televisión]. Estados Unidos: ABC y Showtime.

FROST, M. y LYNCH, D. (Guionista) y LYNCH, D. (Director). (2017, Junio 25). Part 8. [Episodio de serie de televisión] En M. Frost y D. Lynch (Productor), Twin Peaks. Estados Unidos: Showtime.

FROST, M. y LYNCH, D. (Guionista) y LYNCH, D. (Director). (2017, Agosto 20). Part 15. [Episodio de serie de televisión] En M. Frost y D. Lynch (Productor), Twin Peaks. Estados Unidos: Showtime.

FROST, M. y LYNCH, D. (Guionista) y LYNCH, D. (Director). (2017, Agosto 27). Part 16. [Episodio de serie de televisión] En M. Frost y D. Lynch (Productor), Twin Peaks. Estados Unidos: Showtime.

FULLER, B. y DELAURENTIIS, M. (Productor), y FULLER, B. (Creador). (2013-2015). Hannibal [Serie de televisión]. Toronto: NBC.

GARCÍA MARTÍNEZ, A.N. (2018). "La estética del asco. Lo repugnante en la serialidad contemporánea". En La estética televisiva en las series contemporáneas, M. Huerta Floriano y P. Sangro Colón (eds.), 86-102. Valencia: Tirant Humanidades.

GARRONI, E. (1973). Proyecto de semiótica. Barcelona: Gustavo Gili. GENETTE, G. (1989). Figuras III. Barcelona: Lumen.

GILlIGAN, V.; JOHNSON, M. y MACLAREN, M. (Productor) y Gilligan, V. (Creador). (2008-2013). Breaking Bad [Serie de televisión]. Alburquerque: AMC.

GÓMEZ TARÍN, F. J. (2018). “Apocalipsis y melodrama: el tiempo suspendido y la herida final”. L'Atalante. Revista de estudios cinematográficos 25, 41-54.

GROTE, J. y LIGHTFOOT, S. (Guionista) y Medak, P. (Director). (2014, Marzo 14). Hassun. [Episodio de serie de televisión] En B. Fuller (Productor), Hannibal. Toronto: NBC.

HERNÁNDEZ PÉREZ, E. (2013). "Teasing The Audience: Desconfíe de Vince Gilligan". L'Atalante. Revista de estudios cinematográficos $15,42-49$.

HERNÁNDEZ PÉREZ, E. y REVERT GOMIS, J. (2016). “Mad Men, Los 
Soprano y el American way of life. Historia del capital en dos tiempos". Área Abierta 16(3), 17-31.

HITCHCOCK. A. (1954). La ventana indiscreta [Film]. Estados Unidos: Patron Inc.

IORDANOVA, D. (2008). Budding channels of peripheral cinema. New Tork: Blurb.

JINGO, M. (Productor) y KUROSAWA, A. (Director). (1950). Rashomon [Film]. Japón: Daiei Film.

JOHNSON, S. (2005). Everything bad is good for you: how popular culture is making us smarter. London: Penguin.

KINDER, M. (2002). "Hot Spots, Avatars, and Narrative Fields Forever: Buñuel's Legacy for New Digital Media and Interactive Database Narrative". Film Quarterly 55.4, 2-15.

KING, G., MOLLOY, C. y TZIOUMAKIS, Y. (2013). American independent cinema: indie, indiewood and beyond. New York: Routledge.

KLECKER, C. (2013). "Mind-Tricking Narratives: Between Classical and Art-Cinema Narration”. Poetic Today 34 (1-2), 119-146.

KLINGER, B. (2006). Beyond the multiplex: cinema, new technologies and the home. Berkeley: University of California Press.

LEVERETTE, M. (2008). “Cocksucker, Motherfucker, Tits". En It's Not $T V$. Watching HBO in the Post-Television Era, M. Leverette, B. L. Ott y C. L. Buckley (eds.), 123-150. New York: Routledge.

LINDELOF, D.; Perrotta, T.; Berg, P. y Aubrey, S. (Productor) y Lindelof, D. y Perrotta, T. (Creador). (2014-2017). The Leftovers [Serie de televisión]. Estados Unidos: HBO.

LINSON, A. (Productor), y Fincher, D. (Director). (1999). El club de la lucha [Film]. Estados Unidos: Fox 2000 Pictures, Regency Enterprises y Linson Films.

LOBATO, R. (2012). Shadow economies of cinema: mapping informal film distribution. Basingstoke: British Film Institute.

MARZAL-FELICI, J. (1994). Estructuras de reconocimiento y serialidad ritual: el modelo melodrama en los films de David Wark Griffith de 


\section{8-1921. Universitat de València.}

MCCALLUM, R. (Productor) y POTTER, D. (Creador). (1986). El detective cantante [Serie de televisión]. Reino Unido: $\mathrm{BBC}$.

MCLUHAN, M. (1995). Understanding media: the extensions of man. Cambridge: MIT Press.

MEDINA CONTRERAS, J (2018). "Figura y fondo, realidad y ensoñación en Hannibal". En La estética televisiva en las series contemporáneas, M. Huerta Floriano y P. Sangro Colón (eds.), 189-205. Valencia: Tirant Humanidades.

MITTELL, J. (2006). "Narrative Complexity in Contemporary American Television". The Velvet Light Trap 58(1), 29-40.

(2009). "Sites of participation: Wiki fandom and the case of Lostpedia". Transformative Works and Cultures 3 (también en http:// journal.transformativeworks.org/index.php/twc/article/view/118). (2011). "TiVoing childhood: time-shifting a generation's concept of television". En Flow TV. Television in the age of media convergence, M. Kackman et al. (eds.), 46-54. New York: Routledge.

(2013). "Serial Orientations. Paratexts and Contemporary Complex Television". En (Dis)Orienting Media and Narrative Mazes, J. Eckel, D. Olek y C. Piepiorka (eds.), 165-181. Bielefeld: transcript Verlag.

(2014). "Lengthy Interaction with Hideous Men: Walter White and the Serial Poetics of Television Anti-Heroes". En Storytelling in the Media Convergence Age: Exploring Screen Narratives, R. E. Pearson (ed.), 74-92. London: Palgrave Macmillan. (2015). Complex TV: the poetics of contemporary television storytelling. New York: New York University Press.

(2017). "All in the game: The Wire, narración seriada y la lógica del procedimental”. L'Atalante. Revista de estudios cinematográficos 24, 13-25.

MOORE, R. O. (2010). Writing the Silences. Berkeley: University of California Press. 
PALAO ERRANDO, J.A. (2004). La Profecía de la imagen-mundo: para una genealogía del paradigma informativo. Valencia: Ediciones de la Filmoteca.

PALAO-ERRANDO, J.A.; LORIGUILLO-LÓPEZ, A. y SOROLLAROMERO, T. (2018). "Beyond the Screen, Beyond the Story: The Rhetorical Battery of Post-Classical Films". Quarterly Review of Film and Video 35.3, 224-245.

PEARSON, R. (2010). “Observations on Cult Television". En The Cult TV Book, S. Abbott (ed.), 7-18. London, New York: I.B. Tauris.

PIEPIORKA, C. (2013). "You're Not Supposed To Be Confused! (Dis) Orienting Narrative Mazes In Televisual Complex Narrations". En (Dis)Orienting Media and Narrative Mazes, J. Eckel et al. (eds.), 183-202. Bielefeld: transcript Verlag.

PIÑEIRO OTERO, T. (2016). "Intenciones e intersecciones de la música clásica en Hannibal de Bryan Fuller (NBC)". L'Atalante. Revista de estudios cinematográficos 21, 177-189.

RIMMON-KENAN, S. (2002). Narrative Fiction. New York: Routledge.

ROS, E. (2017). "La odisea de Cooper". Jot Down: Contemporary Culture Mag (también en http://www.jotdown.es/2017/09/la-odisea-decooper/ [03/04/2018]).

ROSENFELDT, H.; LARDER, N. y WOOD, T. (Productor) y ROSENFELDT, H. y LARDER, N. (Creador). (2016). Marcella [Serie de televisión]. Reino Unido: ITV.

RUBIO ALCOVER, A. (2011). "Carbon-copy de los años cuerdos. A propósito de Mad Men". L'Atalante. Revista de estudios cinematográficos 11, 12-18.

SHIMPACH, S. (2010). Television in transition: the life and afterlife of the narrative action hero. Malden: Wiley-Blackwell.

SIMONS, J. (2008). "Complex narratives". New Review of Film and Television Studies 6.2, 111-126.

SKOPTSOV, M.L. (2015). "Prophetic Visions, Quality Serials: Twin Peaks' new mode of storytelling". Series - International Journal of 
TV Serial Narratives 1.1, 39-50.

STERRITT, D. (2016). "Puzzle Pictures". Quarterly Review of Film and Video 33.5, 478-481.

THANOULI, E. (2009). Post-classical cinema: an international poetics of film narration. New York: Wallflower Press.

TODD, S. (Productora) y NOLAN, C. (Director). (2000). Memento [Film]. Estados Unidos: Summit Entertainment.

TREEM, S.; LEVI, H.; REINER, J.; OVERMYER, E. y EPSTEIN, A. (Productor), y TREEM, S. y LEVI, H. (Creador). (2014). The Affair [Serie de televisión]. Estados Unidos: Showtime.

VLAMING, J. y FULLER, B. (Guionista) y HUNTER, T. (Director). (2014, Marzo 7). Sakizuke. [Episodio de serie de televisión] En B. Fuller (Productor), Hannibal. Toronto: NBC.

TREEM, S. y LEVI, H. (Guionista) y MYLOD, M. (Director). (2014, Octubre 12). 1. [Episodio de serie de televisión] En S. Treem, H. Levi, J. Reiner, E. Overmyer y A. Epstein. (Productor), The Affair. Estados Unidos: Showtime.

WEINER, M.; HORNBACHER, S.; JACQUEMETTON, A; JACQUEMETTON, M. y LEAHY, J. (Productor), y WEINER, M. (Creador). (2007-2015). Mad Men [Serie de televisión]. Nueva York: AMC.

WILLIAMS, R. (1974). Television: technology and cultural form. London: Fontana.

WILLIAMS, H.; WILLIAMS, J. y STRONG, J. (Productor) y WILLIAMS, H. y WILLIAMS, J. (Creador). (2017-). Liar [Serie de televisión]. Reino Unido: ITV.

Recibido el 29 de abril de 2018.

Aceptado el 6 de septiembre de 2018. 
